\title{
Physics Perspectives of the ALICE experiment at the Large Hadron Collider
}

\author{
Author: \\ M. Masera \\ for the ALICE Collaboration
}

Talk given at the IV International Conference on Physics and Astrophysics of Quark-Gluon Plasma, 26-30 November 2001, Jaipur (India) 


\title{
Physics Perspectives of the ALICE experiment at the Large Hadron Collider
}

\author{
Massimo Masera, for the ALICE Collaboration \\ CERN, CH-1211 Geneve 23, Switzerland. \\ On leave of absence from University of Torino, Italy \\ e-mail: massimo.masera@cern.ch
}

January 25, 2002

\begin{abstract}
The Large Hadron Collider (LHC) under construction at CERN will deliver ion beams up to centre of mass energies of the order of $5.5 \mathrm{TeV}$ per nucleon, in case of lead. If compared to the available facilities for the study of nucleus-nucleus collisions (SpS and RHIC), this represents a huge step forward in terms of both volume and energy density that can be attained in nuclear interactions. ALICE (A Large Ion Collider Experiment) is the only detector specifically designed for the physics of nuclear collisions at LHC, even though it can also study high cross section processes occurring in protonproton collisions. The main goal of the experiment is to observe and study the phase transition from hadronic matter to deconfined partonic matter (Quark Gluon Plasma - QGP). ALICE is conceived as a general-purpose detector and will address most of the phenomena related to the QGP formation at LHC energies: to this purpose, a large fraction of the hadrons, leptons and photons produced in each interaction will be measured and identified.
\end{abstract}

\section{Introduction}

Ultrarelativistic heavy-ion collisions have been studied for fifteen years with a rich experimental programme both at the AGS (Brookhaven National Laboratories) and at the SPS (CERN). It turned out that in these collisions very high energy densities can be reached and there are experimental evidences that in $\mathrm{Pb}-\mathrm{Pb}$ collisions at the SPS a new state of matter in which quarks and gluons are deconfined was formed[1]. The Relativistic Heavy Ion Collider (RHIC) at BNL began its activity in 2000 opening a new horizon for studying nucleus-nucleus interactions. The centre of mass energy $(\sqrt{s}=200 \mathrm{GeV}$ per nucleon) is one order of magnitude higher than what can be achieved at the SPS hence the temperature reached in the collision should well exceed the critical value for the phase transition to the QGP. The amount of available experimental results from RHIC is already impressive[2]. The next step towards really high energies will be made by the Large Hadron Collider (LHC) that will be ready for physics in the year 2006. The available centre of mass energy will be $5.5 \mathrm{TeV}$ per nucleon with $\mathrm{Pb}$ beams, 27.5 times higher if compared to RHIC. The ALICE (A Large Ion Collider Experiment) experiment is focused on heavy-ion physics at the LHC, even though it is able to address relevant 


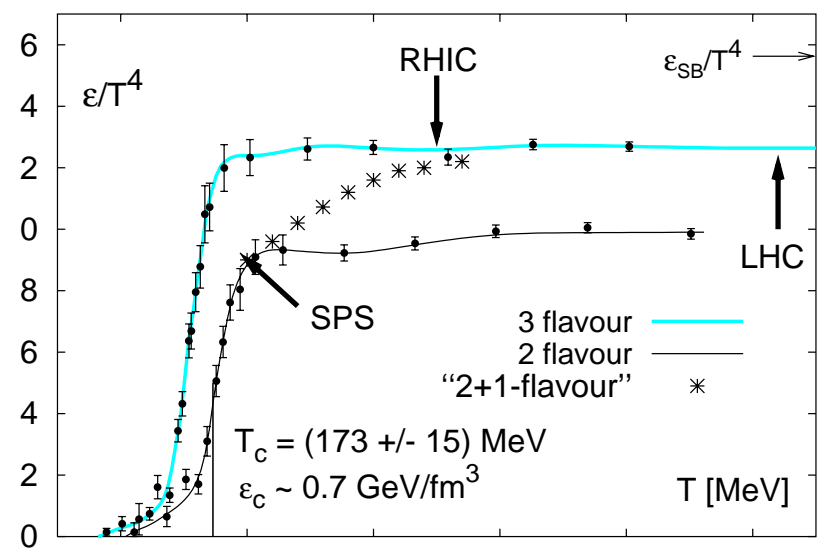

Figure 1: Lattice QCD phase transition (F.Karsch in [11])

high cross section $\mathrm{p}-\mathrm{p}$ phenomena[3]. Its physics perspectives, as far as nucleus-nucleus interactions are concerned, will be presented in this paper.

\section{A Heavy-Ion experiment at the LHC: why?}

Some of the parameters characterizing a nucleus-nucleus collision for SPS, RHIC and LHC are listed in Table 1 (adapted from[5]) which has to be considered as an orderof-magnitude comparison tool. The extrapolation from SPS to RHIC was far from being obvious; for instance many predictions for particle multiplicity have been done starting from several models with different assumptions on the QCD dynamics leading to results spanning over a rather wide range $\left(600<d N_{c h} / d \eta<1500\right)$ of values with a general tendency to overestimate the multiplicity actually observed at RHIC[4]. Even

Table 1: Comparison of parameters characterising A-A collisions. See text for details.

\begin{tabular}{|l|r|r|r|}
\hline $\begin{array}{l}\text { Parameters for central } \\
\text { A-A collisions }\end{array}$ & SPS & RHIC & LHC \\
\hline \hline$\sqrt{s} / \mathrm{A}(\mathrm{GeV})$ & 17 & 200 & 5500 \\
\hline$d N_{c h} / d \eta$ & 400 & 650 & $2 \div 810^{3}$ \\
\hline$\epsilon \quad\left(\mathrm{GeV} / \mathrm{fm}^{3}\right)$ & 2.9 & 25 & 200 \\
\hline$V_{f}\left(\mathrm{fm}^{3}\right)$ & few $10^{3}$ & few $10^{4}$ & few $10^{5}$ \\
\hline$\tau_{Q G P \quad(\mathrm{fm} / \mathrm{c})} \quad(\mathrm{fm} / \mathrm{c})$ & 1 & $2 \div 4$ & $\geq 10$ \\
\hline$\tau_{0}^{Q G P} \quad$ & 1 & 0.2 & 0.1 \\
\hline
\end{tabular}

though the data from RHIC are useful to tune and possibly rule out models, they do not reduce too much the uncertainties on the predictions for multiplicity at LHC, which vary from slightly below 2000 up to 8000 charged particles per unit of rapidity. The particle production in nucleus-nucleus collisions scales with the number of the participants at SPS energies, where soft processes dominate. The role of hard processes, which are dependent on the number of collisions $\left(N_{\text {coll }} \propto A^{4 / 3}\right)$ also increases with increasing $\sqrt{s}$ and it is expected to be dominant at LHC. Another factor that contributes to enhance the multiplicity at high energy is related to the fact that the structure functions increase steeply with decreasing $x_{B J}$. This leads to an enhanced number of partons taking part in 
the interaction. The effect of this increase in the number of partons is somewhat reduced by the shadowing. It should be noted that at LHC, for moderate $Q^{2} \sim 10 \mathrm{GeV}^{2}$, we will reach $x_{B J}$ values in the range of $510^{-4}$ in the central region and down to almost $10^{-5}$ in the forward region (ALICE muon spectrometer), where shadowing effects are strong. Moreover, phenomena like parton saturation and jet quenching should be particularly relevant at the LHC. The uncertainties on these processes do not allow to make a reliable evaluation of the multiplicities. The value $d N_{c h} / d \eta=8000$ is therefore used in the simulations for ALICE as an upper limit at which the detector must still be functional.

The thermalization time $\left(\tau_{0}^{Q G P}\right)$ decreases with increasing $\sqrt{s}$ essentially for two reasons. On one hand the formation time of partons is inversely proportional to the tranverse momentum which, in turn, increases slowly with $\sqrt{s}$ and on the other hand higher parton densities (mainly gluons at LHC) lead to a higher interaction rate. As a consequence the temperature and the energy density are expected to be very high at LHC. In Fig. 1 the temperature dependence of $\epsilon$ as computed in lattice QCD is shown under different assumptions: two and three light quarks and two light quarks plus one heavier quark (s). In Fig. 1 the expected behaviour is indicated by the stars: at temperatures $T \simeq T_{c}=173 \pm 15 \mathrm{MeV}$, the quark s does not contribute to the thermodynamics due to its mass whereas at high temperature the energy density is essentially given by 3 light flavour QCD. At the LHC, the colliding system is expected to be well within the plateau at $T \sim 3 T_{c}$ with a vanishing baryochemical potential at $y \sim 0$. In this regime of $\mu_{B}<<T$ the equation of state approaches the better understood case of $\mu_{B}=0$. The ideal limit of a Stefan-Boltzmann gas of quarks and gluons is not reached even at the highest temperatures indicating that non perturbative effects are still relevant. The high temperature reached at LHC extends also the lifetime and the volume of the formed QGP since it has to expand while cooling down to the freeze-out temperature, which is independent of the energy of the collision. Longer lifetime and volume imply that signals coming from the plasma phase should be strongly enhanced at LHC.

At LHC energies, perturbative QCD can be applied: hard processes with high momentum transfers are already significant at RHIC and will be dominant at LHC. The hard signals, namely heavy flavours and high $p_{T}$ jets, are created at early times and probe the medium formed after the collision. The study of charmonium states, initiated at the SPS, will be extended at LHC to the $\Upsilon$ family since the production cross section will be sufficiently high and the melting point expected for these resonances will be exceeded at the LHC. A direct measurement of open charm will be feasible after indirect hints of its possible enhancement already at SPS energy. The open charm detection will provide also a normalization for the $J / \Psi$. Finally, the relative abundance analysis of hadrons via particle ratios will be extended to charmed particles. The energy loss of high $p_{T}$ partons can be studied via jet measurements: the expected jet quenching due to this phenomenon is in fact a probe that can be fully exploited at LHC energies. The possibility of measuring several observables on a event-by-event basis increases with the number of produced particles so at LHC the event-by-event physics will be a precision tool suitable for searches of non statistical fluctuations which are expected to occur at the phase transition.

\section{The ALICE experiment}

ALICE is the only experiment at LHC especially conceived to study the physics of strongly interacting matter and the QGP. For this reason it has to cover as much observables as possible. It is operated by an international collaboration formed by more than 900 physicists from 77 institutions of 28 different countries. Few weeks per year 


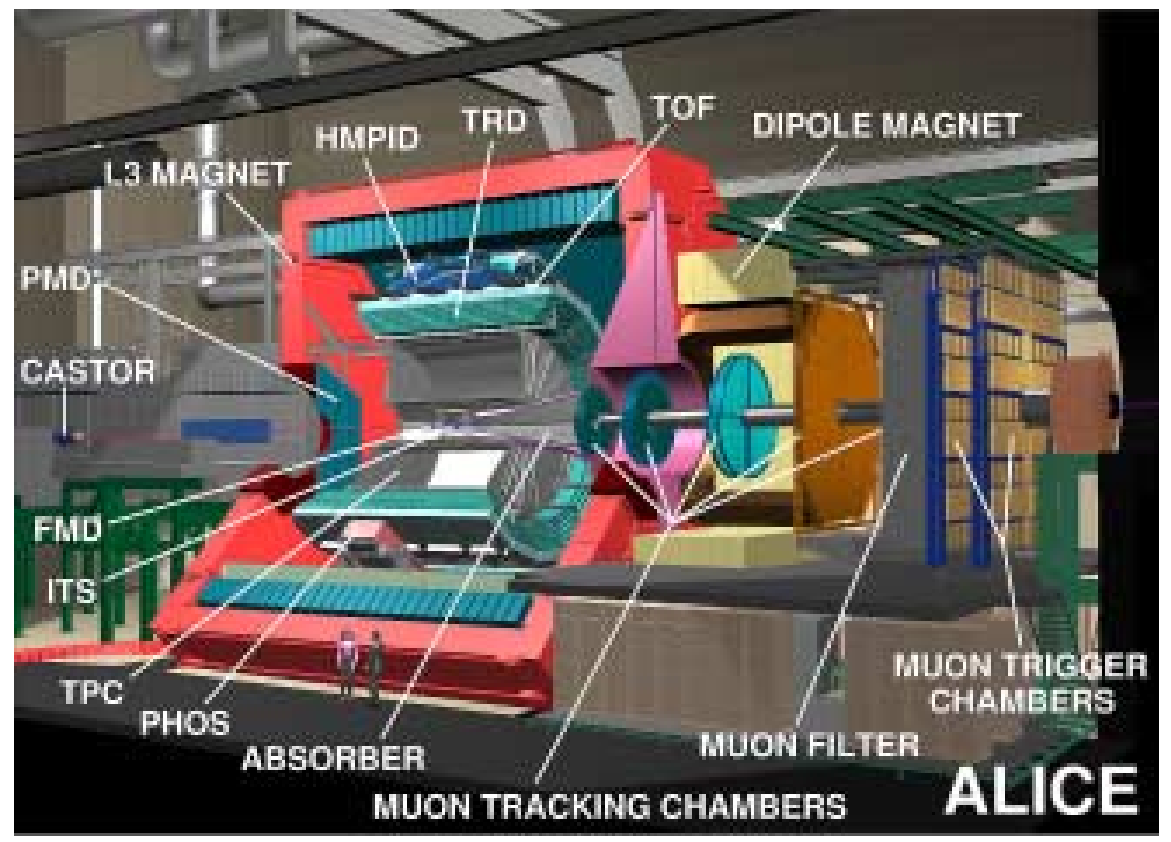

Figure 2: The ALICE experimental apparatus

$\left(\sim 10^{6} \mathrm{~s}\right)$ will be devoted to nucleus-nucleus physics at a luminosity that is limited to $\mathcal{L}=10^{27} \mathrm{~cm}^{-2} \mathrm{~s}^{-1}$ for $\mathrm{Pb}-\mathrm{Pb}$. With a $\sigma_{I}=8 \mathrm{~b}$, the expected number of interactions per second is $\sim 8000(\sim 10 \%$ being central $)$.
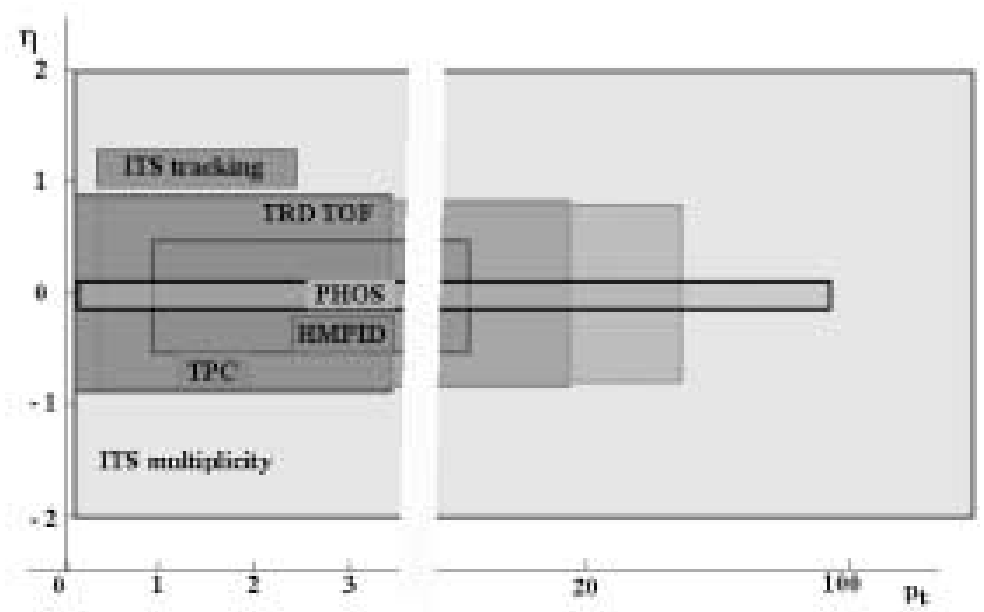

Figure 3: ALICE central barrel detectors: acceptances

\subsection{Observables}

The ALICE physics goals can be summarized, albeit not exhaustively, as follows:

- Global event features. Multiplicities, very forward energy flow (0 degree) and rapidity distributions allow to determine the centrality, the number of participants in the interaction and to specify the initial energy density.

- The geometry and space-time evolution of the emitting source. The space-time structure of the collision fireball will be studied with two-particle momentum cor- 
relations.

- Degrees of freedom as a function of temperature. Quantities related to the dynamical evolution of the hadronic phase like $p_{T}$ spectra and particle ratios of identified hadrons $(\pi, \eta, \omega, \phi, p, K, \Lambda, \Xi, \Omega)$ and direct photons will be measured.

- Non statistical fluctuations and critical behaviour. This topic will be addressed by event-by-event analyses, including some spectra and particle ratios. Distortions of $N_{\gamma}-N_{\text {charged }}$ correlations will be suitable for the detection of Disoriented Chiral Condensates (DCC). Searches for anomalies like the Centauro events will be also performed.

- Chiral symmetry restoration. It will be searched through the study of the resonance decays.

- Collective effects: elliptic and directed flow will be investigated by various sub detectors.

- Hard probes. Open charm and beauty, high $p_{T}$ spectra, jets and jet quenching will be measured. Spectroscopy of the $J / \Psi$ and $\Upsilon$ families will provide a tool particularly sensitive to deconfinement.

These physics goals imply a good particle identification capability of both hadrons and leptons over a wide acceptance and an extended $p_{T}$ domain. As a difference from the other LHC experiments, the sensitivity to low $p_{T}$ particles is relevant for many of the physics issues addressed by ALICE. Therefore the main experimental challenge consists in tracking and identify virtually all the produced stable particles in an environment in which these particles could be as many as 8000 per unit of rapidity.

\subsection{The experimental apparatus}

The experimental layout is represented in Fig. 2. The relatively low interaction rate expected with nuclear beams together with the high particle density lead to a solution based on a Time Projection Chamber (TPC) used for tracking in the central rapidity region. This detector, which is able to sustain the foreseen rate has a high granularity over a wide region. It extends longitudinally from $-2.5 \mathrm{~m}$ to $2.5 \mathrm{~m}$ and radially from $90 \mathrm{~cm}$ up to $250 \mathrm{~cm}$ in order to have a $d E / d x$ resolution $<10 \%$ for electron identification. The design and the choice of the gas are optimised for a good double-track resolution. The total number of channels is $5.710^{6}$ feeding the DAQ with an amount of information of $\sim 60 \mathrm{MB}$ for a central event, corresponding to 12000 tracks. The pseudorapididity coverage (see Fig. 3) of the TPC and the other detectors of the central barrel is $|\eta|<0.9$, which is adequate for studying particle ratios, $p_{T}$ spectra and HBT correlations.

The magnetic field necessary for momentum measurements is provided by the magnet of the L3 experiment: it generates a weak solenoidal field $(B<0.5 T)$ over the whole region of the barrel. This choice allows a low momentum cut-off $(\sim 100 \mathrm{MeV} / \mathrm{c})$ and, at the same time, a good tracking efficiency and momentum resolution.

The six layers of silicon detectors forming the Inner Tracking System (ITS) are used for track reconstruction close to the vertex. The innermost layer is located at a radius $r=4 \mathrm{~cm}$, only $1 \mathrm{~cm}$ from the beam pipe, while the sixth layer is located at $r=44 \mathrm{~cm}$. The main goal of the ITS is to reconstruct secondary vertices from hyperons, $K_{0}^{S}$ and charmed mesons decays, hence the vertexing resolution must be $<100 \mu \mathrm{m}$. This constraint, together with the high track density, led to the choice of detectors that provide a bidimensional information such as silicon pixels in the first two layers and silicon drift detector for the third and fourth layers. The last two layers, due to the relatively large surface to be equipped, employ double sided silicon strip detectors. The ITS is also 


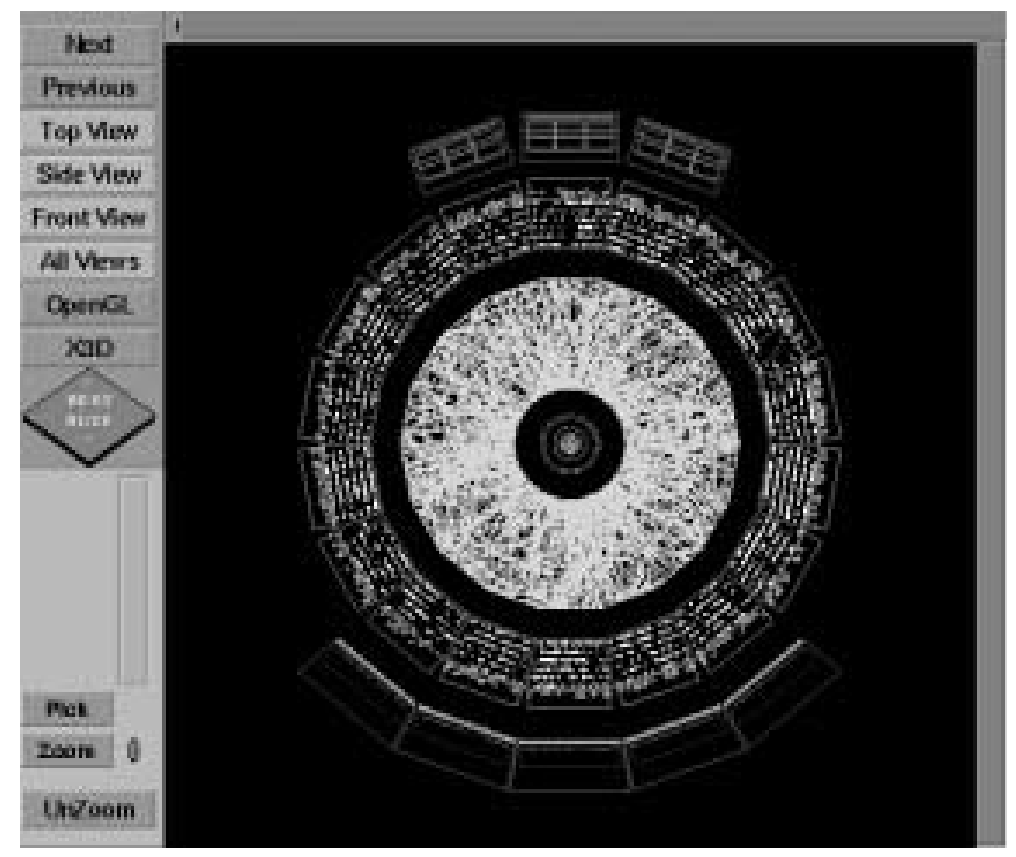

Figure 4: AliRoot event display. Particles are generated with a polar angle $\theta$ in the range $85<\theta<95$ with a density corresponding to the maximum foreseen value. Starting from the beam axis, ITS, TPC, TRD and TOF are displayed. PHOS and HMPID are below and above the beam axis respectively

needed for low momentum particle identification through $d E / d x$ measurements in layers 3 to 6 and to improve the momentum resolution at large momenta.

As it is illustrated in Fig. 3, the particle identification (PID) is performed by using several detectors exploiting all methods available to this purpose, since PID is one of the key issues of this experiment. A Time Of Flight (TOF) detector, located in the barrel at $3.7 \mathrm{~m}$ from the beam axis, identifies $\pi^{ \pm}, K^{ \pm}$, protons and contributes to $e / \pi$ rejection. The TOF is made with multi-gap resistive plate chambers with an intrinsic time resolution $<100 \mathrm{ps}$. The extension of the hadron identification into the semi-hard region (good $p, K$ separation for $p_{T}<5 \mathrm{GeV} / \mathrm{c}$ ) is achieved, with a reduced acceptance, by means of a proximity focusing RICH detector (HMPID) located at $\sim 4.5 \mathrm{~m}$ from the beam axis. The electron identification in the barrel is complemented at high $p_{T}$ $\left(p_{T}>1 \mathrm{GeV} / \mathrm{c}\right)$ by a dedicated Transition Radiation Detector (TRD) consisting of six layers of radiator foil stacks followed by Time Expansion Chambers, providing a $\pi$ rejection $>50$ at the maximum occupancy. As a fast response detector, it can be used for triggering on high momentum electrons $(p>3 \mathrm{GeV} / \mathrm{c})$.

Detection of direct photons and of neutral mesons $\left(p_{T}>1 \mathrm{GeV} / \mathrm{c}\right)$ like $\pi^{0}$ and $\eta$ through their $2 \gamma$ decay mode is achieved with a single arm, high resolution electromagnetic calorimeter (PHOS) placed $4.6 \mathrm{~m}$ from the vertex. The detector is made by 17920 crystals of $\mathrm{PbWO}_{4}$ scintillator grouped in 5 modules. The choice of this material is due to the requirement of a Molière radius as small as possible, together with a good light output.

The muon channel will be studied by a dedicated forward muon spectrometer in the pseudorapidity range $2.4<\eta<4$. It consists of a hadronic absorber $\simeq 10 \lambda_{I}$ thick starting close to the interaction region in order to reduce the combinatorial background from $\pi^{ \pm}$and $K^{ \pm}$decays. The absorber, which is composite, is designed in order to 


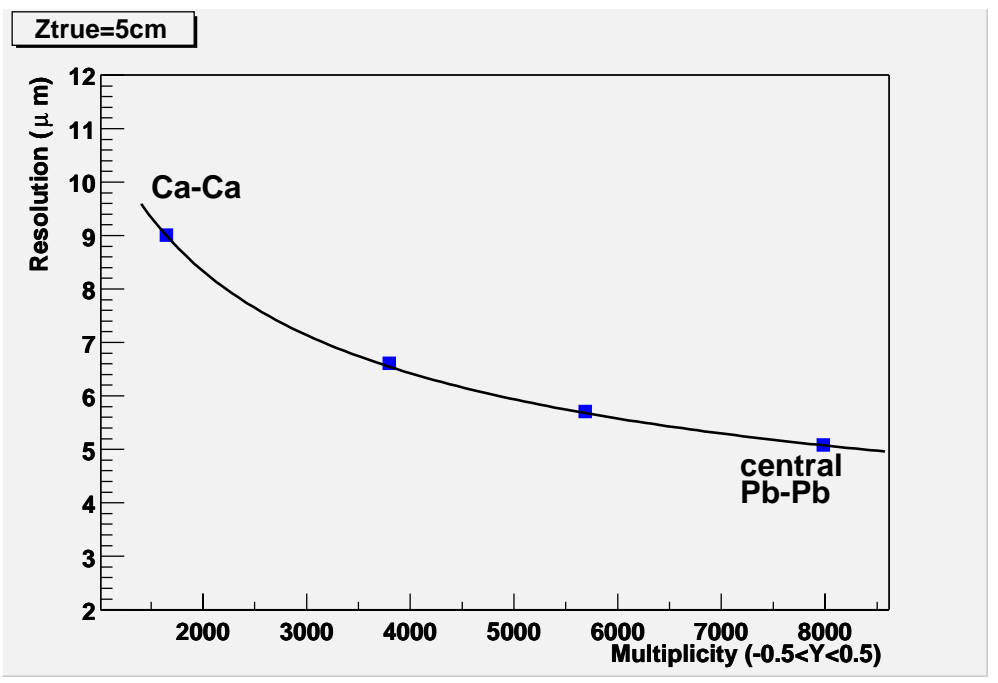

Figure 5: Resolution on the primary vertex $\mathrm{Z}$ coordinate as a function of the multiplicity in the central unit of rapidity. The full line is a fit with the function $\sigma_{z}=B+A / \sqrt{d N_{c h} / d \eta}$ with $A=292$ and $B=1.8$

minimize both the multiple scattering and the leakage. Muons are then tracked in a $0.7 \mathrm{~T}$ dipole magnet (for an overall $3 \mathrm{Tm}$ ) by means of 10 Cathode Pad Chambers. A second absorber, placed at the end of the spectrometer, followed by four planes of Resistive Plate Chambers is used for muon identification and triggering. The main goal of the spectrometer is the study of the charmonium and bottomium states. A mass resolution of $100 \mathrm{MeV} / \mathrm{c}^{2}$ at the $\Upsilon$ mass is required for this purpose[6].

The apparatus is complemented by several smaller detecting systems placed at forward rapidities: the impact parameter of the collision is measured by a set of 4 small calorimeters to be installed in the machine tunnel at both sides of the interaction region at a distance of $\sim 100 \mathrm{~m}$. Electromagnetic and transverse energy at high rapidity $(5.3<\eta<7.3)$ are measured by the CASTOR calorimeter. The pseudorapidity distribution of charged particles will be determined by the Forward Multiplicty Detector for $-5.1<\eta<-1.7$ and $1.7<\eta<3.4$. Searches for non statistical fluctuations in the ratio of photons and charged particles, the study of collective flow and the determination of the interaction plane will be carried out by the Photon Multiplicity Detector (PMD)[7]. Finally, the time of the event will be measured by the T0 and V0 detectors. A complete description of the current status of the apparatus can be found in[8]

\subsection{Expected performances}

The evaluation of the physics performances of ALICE is a process that began with the preparation of the Technical Proposal[9] of the experiment, continued with the Technical Design Reports[10] of the single subsystems and it is the goal of a dedicated report (PPR) that is in preparation now[11]. The most recent summaries can be found in [12] for hadronic signals and in [13] for heavy quark measurements. The developement of the offline framework necessary for the PPR represents a major challenge for the Collaboration, given the complexity of the apparatus and the large amount of information involved. Since 1998 a new Object Oriented framework, AliRoot[14] is being developed starting from ROOT[15], which is a $\mathrm{C}++$ package written for high energy physics. AliRoot provides an interface with event generators like Pythia[16] and Hijing[17], with simulation programs like GEANT3, FLUKA and GEANT4. Relic Fortran code is wrapped in C++ 


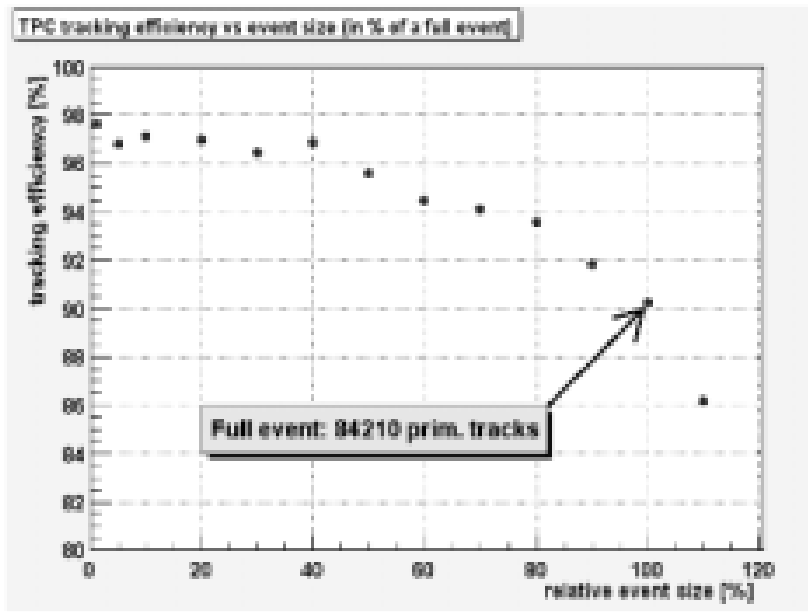

Figure 6: Tracking efficiency in the TPC as a funtion of the size of the event.

user interfaces, so all new code has been written in $\mathrm{C}++$. Reconstruction and analysis phases are also done in the AliRoot environment. This package, which is in continuous evolution, has been already used for most of the simulations needed for the Technical Design Reports. A large scale simulation, spread over several computing centres around the world started in October 2001 for the preparation of the PPR[11].

In Fig. 4, a $10^{\circ}$ polar slice of a central $\mathrm{Pb}-\mathrm{Pb}$ event is displayed: it is clear that tracking in the central barrel is the real challenge for ALICE, since most of the physical observables addressed by this experiment depend on the tracking capabilities of the apparatus.

The status of tracking in the barrel will be reported here; the reader is referred to the papers mentioned above for a complete report.

A tracking efficiency of the order of $90 \%$ for particles with transverse momenta greater than $100-150 \mathrm{MeV} / \mathrm{c}$ and a momentum resolution $\delta p / p \sim 1-2 \%$ are the design requirements for the reconstruction in the TPC and the ITS. The method now implemented in AliRoot is based on the Kalman filter[18]: it has the advantage of being a track finding and a track fitting method at the same time. Furthermore, there is no need of having a track model and there is no need of manipulating all the measured points belonging to the same track together, since the Kalman filter is a local tracking method. Incorrect space points, due to noise or to failures of the cluster finding algorithms can be rejected during the track finding without using an additional track fitting pass, which would be needed with global tracking approaches. Each track is represented at a given point by 5 numbers and the size of the covariance matrix to be handled is $5 \times 5$ in contrast to what happens with a global method in which matrices of the size of the number of measured points must be inverted during fit. Stochastic effects are naturally taken into account in the filtering process and the propagation of tracks from a subdetector to another is relatively easy. The price to pay is that this technique cannot be used with raw data, so a cluster finding algorithm must be applied in advance and this is not generally an easy task. For instance in the inner sectors of the TPC, the occupancy is $\sim 40 \%$ and some clusters may be lost or displaced. Moreover, the track finding has to be initiated with track segments which have to be preliminarily determined. This operatiorn is called seeding. In ALICE, the track seeding can be performed by combining the clusters on two predetermined pad rows of the TPC so the reconstruction in the entire barrel starts from this detector. 


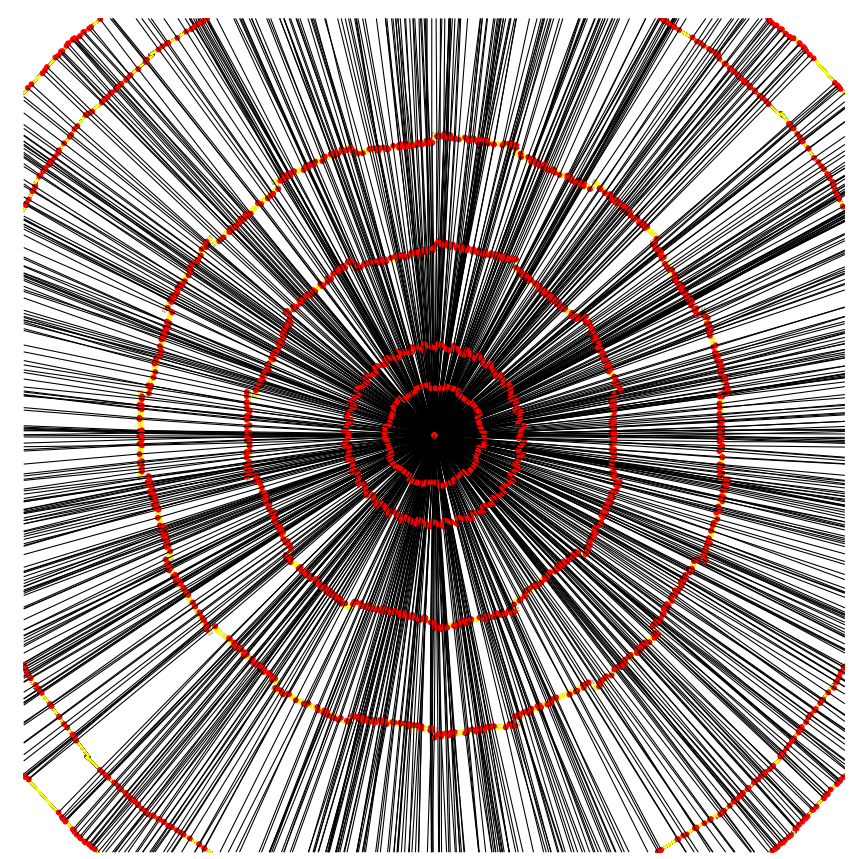

Figure 7: Reconstructed tracks in ITS $\left(p_{T}>1 \mathrm{GeV} / \mathrm{c}\right)$

To this purpose the vertex position in the interaction region must be known, even though the vertex constraint for seeding is not applied strictly in order to be able to reconstruct also secondary particles. As illustrated in Fig. 5 the position of the primary vertex can be measured with very good accuracy by means of the first two high granularity layers of the ITS: the algorithm is very simple and makes use of the correlation among silicon pixels belonging to the two different layers. The higher is the multiplicity the better is the accuracy. The resolution in the $r-\varphi$ plane is of the order of $25 \mu \mathrm{m}$. The resolution is more than adequate for the tracking procedures and this technique can still be applied in the case of $\mathrm{p}-\mathrm{p}$ events, where the resolution is $\sim 150 \mu \mathrm{m}$. The tracking efficiency in the TPC is shown in Fig. 6 as a function of the generated particle multiplicity. The efficiency is computed with respect to the number of particles which produce a sufficient number of clusters in the TPC (trackable particles). A relative event size of $100 \%$ corresponds to a central multiplicity density of 8000 charged particles. In this case the overall number of particles is more than 80000 and the efficiency is still $90 \%$. The reconstruction in the ITS is carried out starting from the tracks actually found in the TPC. The method adopted in the ITS is not purely local, since a vertex constraint is applied in order to cope with the track density. A track is found when there is a matching on all the 6 layers of the ITS. In Fig. 7, reconstructed tracks in the ITS with a transverse momentum higher than $1 \mathrm{GeV} / \mathrm{c}$ are displayed: the first five silicon layers are clearly visible. In Fig. 8, the distribution of the coordinate along the beam axis of the reconstructed vertex after tracking in the TPC+ITS is shown. The distribution width is $\sim 50 \mu \mathrm{m}$. With this resolution, the detection of secondary vertices is possible, also in cases in which the $c \tau \simeq 120 \mu \mathrm{m}$ as for $D^{0} \rightarrow K^{-} \pi^{+}$. This decay channel of $D^{0}$ meson is actually the most promising one to study hadronic charm production in ALICE[13]. In Fig. 9 both the resolution in azimuth and $p_{T}$ for a central $\mathrm{Pb}-\mathrm{Pb}$ event are shown. With a magnetic field $B=0.4 T$ the $p_{T}$ and $\varphi$ resolutions are $\sim 1 \%$ and $\sim 0.5 \mathrm{mrad}$ respectively. These are very preliminary evaluations that must be reassessed by using the large amount of simulated data now available. In Fig. 10 reconstructed $p_{T}$ spectra are shown. The event generator used is a parametrization of Hijing and only $K$ and $\pi$ 


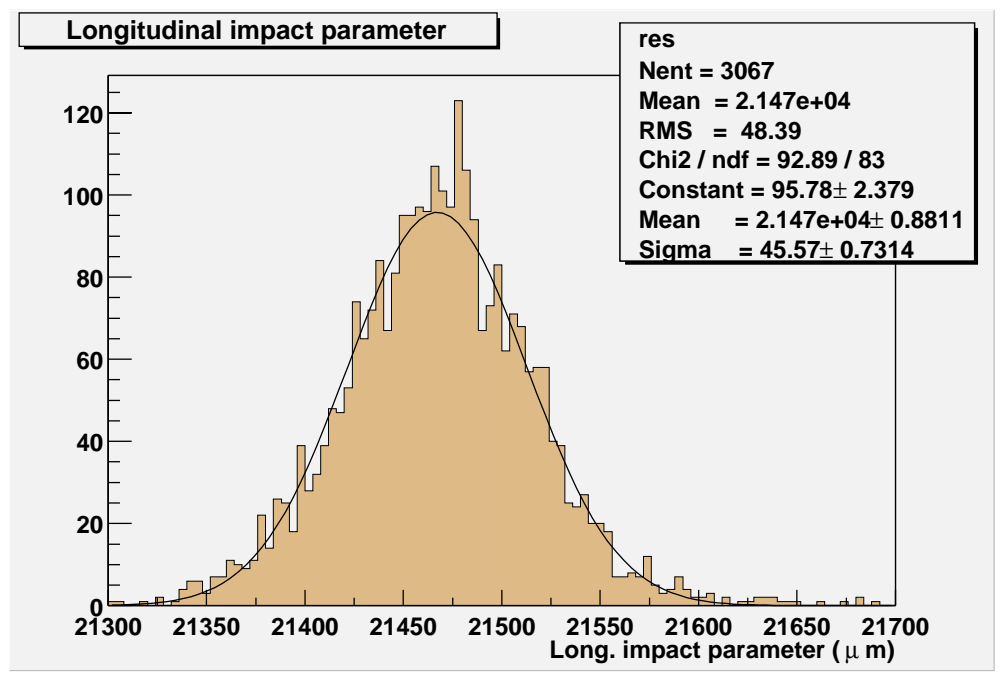

Figure 8: Distribution of the $Z$ coordinate of the reconstructed tracks, extrapolated to the primary vertex

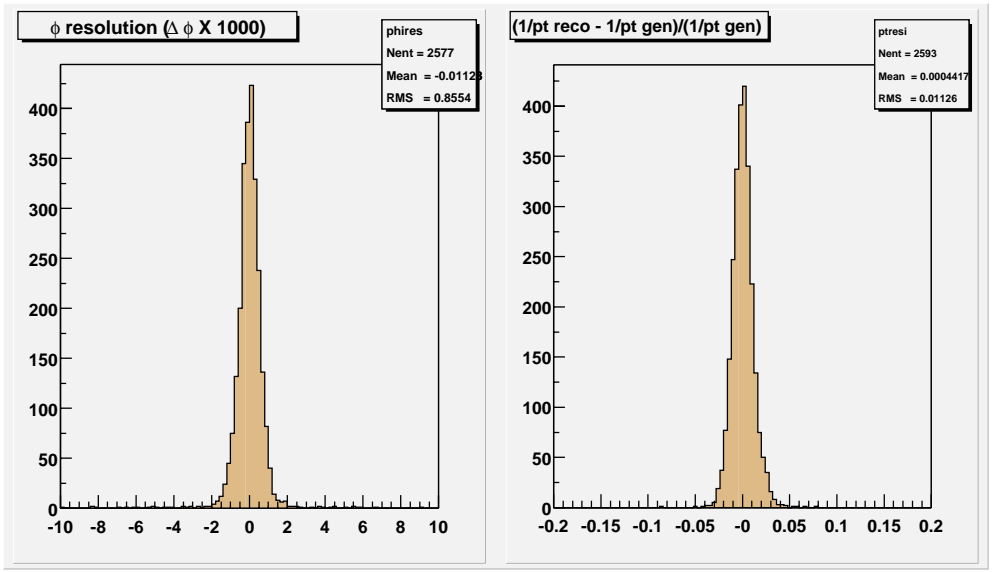

Figure 9: Resolutions in azimuth and $p_{T}$ for tracking in TPC+ITS

were actually generated. When comparing reconstructed spectra with generated ones, it turns out that the reconstruction does not introduce distortions. The tracks obtained in TPC+ITS are then backpropagated to the outer barrel detectors. For instance, in Fig. 11 the invariant mass spectrum of dielectron pairs from $\Upsilon$ decay is plotted. The invariant mass has been obtained in the TRD after a complete tracking in the barrel and the application of a trigger momentum cutoff.

The good tracking performance in the barrel is necessary also for studying jets in ALICE. Usually jets are reconstructed by means of electromagnetic and hadronic calorimeters, but in ALICE there are no hadronic calorimeters and the acceptance of PHOS is limited. Since particles with $p_{T}$ up to $\sim 40 \mathrm{GeV}$ can be measured with good resolution, jets can be found by looking for the leading particle. A jet finding procedure with leading particles is presently under test. Reconstructed particles in the TPC with $p_{T}>4 \mathrm{GeV} / \mathrm{c}$ are used as a seed for jet finding. All the particles with $p_{T}>2 \mathrm{GeV} / \mathrm{c}$ belonging to a cone of width $\Delta R=\sqrt{\Delta \varphi^{2}+\Delta \eta^{2}}<\Delta R_{0}$ centered on the "seed" particle are associated to the jet $\left(\Delta R_{0}=0.7\right.$ in the present tests). The momentum vectors of the found particles are summed up. This procedure is repeated iteratively till no more seeds are left. The first results are good and a trigger for high $p_{T}$ jets based on the TRD is under study. 


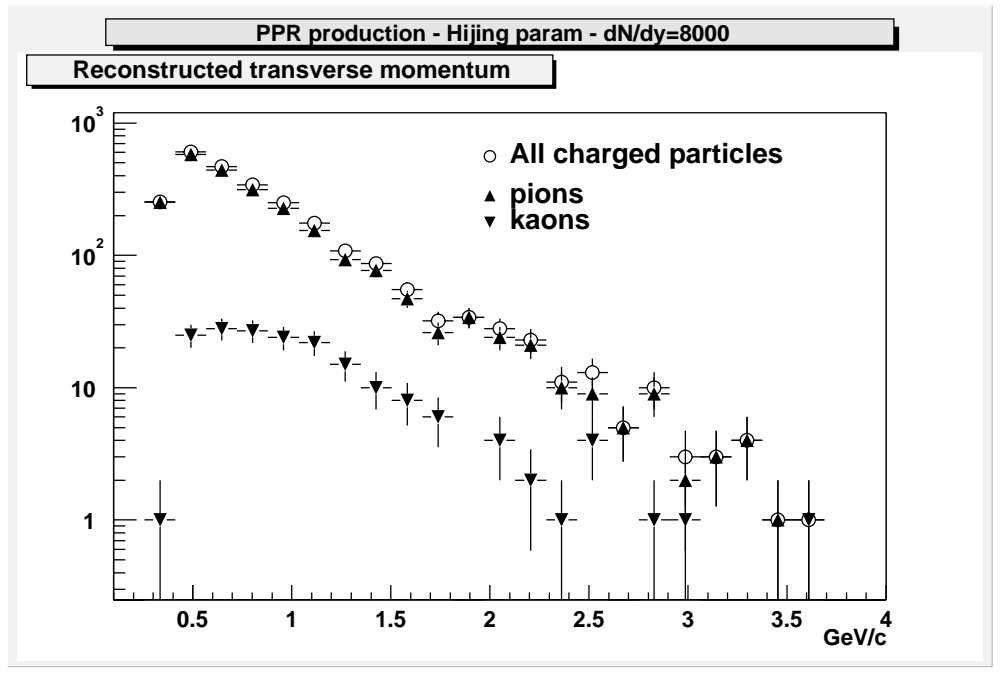

Figure 10: Reconstructed $p_{T}$ in ITS+TPC. The event has been generated with a Hijing parametrization with $d N_{c h} / d \eta=8000$

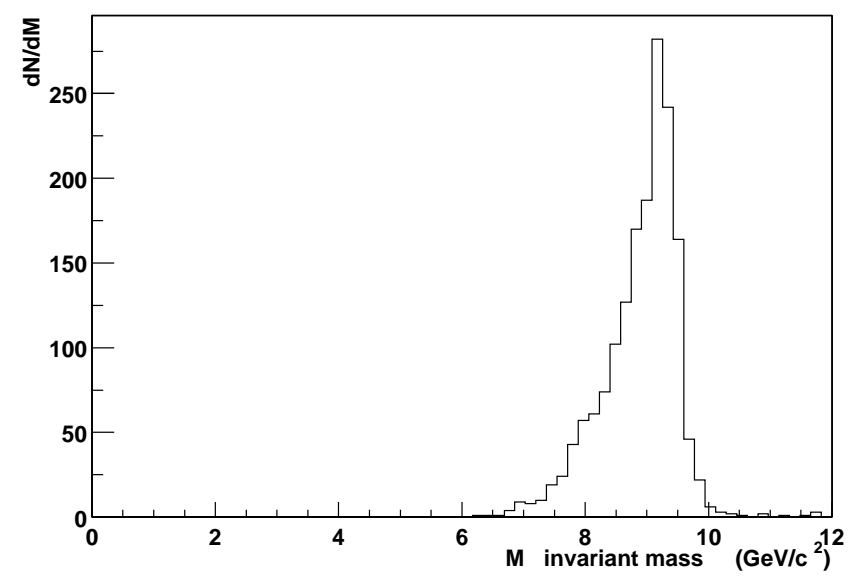

Figure 11: Invariant mass $e^{+} e^{-}$distribution in case of $\Upsilon \rightarrow e^{+} e^{-}$

Another possibility of studying jets in ALICE is to use the PHOS to tag jets originating from $\gamma q$ and $\gamma g$ topologies: in these cases an energetic photon in the PHOS is associated to a hadronic jet in the TPC.

\section{Conclusions}

With the advent of the LHC it will be possible to study the QGP well above the energy density of the phase transition. There will be a sizeable improvement in all the parameters related to the quark gluon plasma formation and to the possiblity of studying it on a more stable theoretical ground. The ALICE experiment just ended its research and developement phase and entered the construction phase. The design of the apparatus is now well defined and the available simulation and analysis tools have reached a high level of accuracy and reliability: the Collaboration is making a great effort to study in detail the physics capabilities of the experiment. The results obtained so far are confirming that ALICE will be able to exploit the possibilities of studying strongly interacting matter available at the LHC. 


\section{References}

[1] U. Heinz and M. Jacob, nucl-th/0002042, 16/02/2000

[2] See contributions to these Proceedings of B.Back (PHOBOS), I.Bearden (BRAHMS), H.Caines (STAR) and A.Drees (PHENIX)

[3] J.P. Revol, these Proceedings

[4] K. J. Eskola, Nucl. Phys. A698 (2002) 78 [hep-ph/0104058]

[5] J. Schukraft, Nucl. Phys. A698 (2002) 287 [CERN-ALI-2001-009]

[6] P. Crochet, these Proceedings

[7] B. Mohanty, these Proceedings; S. Raniwala, these Proceedings

[8] P.Giubellino for the ALICE Collaboration, Proc. of the III International Symposium on LHC Physics and Detectors, Chia, Italy, October 25-27, 2001

[9] ALICE Collaboration, Technical Proposal, CERN/LHCC/95-71 (1995); Addendum to the Technical Proposal CERN/LHCC/96-32 (1996)

[10] ALICE Collaboration, Technical Design Reports. HMPID: CERN/LHCC/9819 (1998); PHOS: CERN/LHCC/99-4 (1999); ZDC: CERN/LHCC/995; ITS: CERN/LHCC/99-12; MUON: CERN/LHCC/99-22 (1999) and CERN/LHCC/2000-046 (2000); PMD: CERN/LHCC/99-32 (1999); TPC: CERN/LHCC/2000-001 (2000); TOF: CERN/LHCC/2000-012 (2000); TRD: CERN/LHCC/2001/021 (2001)

[11] Alice Collaboration, Physics Performance Report, in preparation

[12] P.Foka for the ALICE Collaboration, Proc. of the III International Symposium on LHC Physics and Detectors, Chia, Italy, October 25-27, 2001

[13] P. Crochet for the ALICE Collaboration, Proc. of the III International Symposium on LHC Physics and Detectors, Chia, Italy, October 25-27, 2001

[14] AliRoot: source code and documentation available at http:://AliSoft.cern.ch/offline

[15] Root: source code and documentation available at http:://root.cern.ch

[16] T. Sjöstrand, Comput. Phys. Commun. 82 (1994) 74; T. Sjöstrand, hep-ph/0108264 (2001)

[17] X.N. Wang and M. Gyulassy, Phys. Rev. D44 (1991) 3501; X.N. Wang and M. Gyulassy, Comput. Phys. Commun. 83 (1994) 307

[18] P. Billoir, Nucl. Instrum. Methods 225 (1984) 352; P. Billoir, S. Qian, Nucl. Instrum. Methods A294 (1990) 219; B. Batyunya et al, ALICE Internal Note ALICE/97-24 (1997) 